\title{
Sulphone Resistance and Its Implications
}

\author{
L. M. HOGERZEIL \\ Victoria Hospital, Dichpalli, India
}

\begin{abstract}
The most important factors in the emergence of sulphone resistance are low dose therapy and irregular treatment with dapsone. Measures are discussed how to increase treatment in depth and effectiveness.
\end{abstract}

\section{Introduction}

Around 1961 the first cases of sulphone-resistant leprosy were detected at Carville, U.S.A., and Sungei Buloh, Malaysia. At first leprosy workers were slow to appreciate the serious implications of these findings. During the 9th International Leprosy Congress in London (1968) only 2 papers (Rees, 1968; Pearson et al., 1968) out of 236 were devoted to sulphone resistance in leprosy and the 10th Congress in Bergen (1973), with 7 papers out of 378 paying full attention to the subject, did hardly any better. Since then detailed reports from all over the world have made it clear that sulphone resistance is rapidly on the increase, in some countries at the rate of $2-3 \%$ per annum in lepromatous patients. Although by now most leprosy workers are aware of the existence of sulphone resistance there seems to be a curious reluctance to accept this unpleasant truth and act upon it. In many places policies of control programmes and treatment of individual patients continue in much the same way as before, perhaps in the hope that one day the problem of resistance, simply by ignoring it, will have disappeared of its own account.

\section{Low Dose Therapy}

The main reasons for the emergence of sulphone resistance appear to be low dose therapy and irregular treatment with DDS. Low dose therapy was inspired by the conception that there is a direct connection between the dosage of dapsone and the occurrence of reactions. Hence treatment is usually started with a small dose of DDS, e.g. $2 \frac{1}{2}-5 \mathrm{mg}$ daily, and this is slowly increased over a number of months to 50-100 mg daily. But as soon as reactions occur the dosage is reduced or treatment stopped, with the result that many lepromatous patients continue for months or even years on a dosage of DDS insufficient to prevent the occurrence of sulphone resistance. Nowadays a growing number of leprologists are of the opinion that reactions are not due to treatment with DDS and that as a consequence the dosage need not be reduced during episodes of reaction. 
However this may be, while weighing against each other the risks of sulphone resistance and of reactions it is evident that the former is the graver and should be tackled first. This means that all patients should be started ab initio on full dose DDS $\left(1 \frac{1}{2}-2 \mathrm{mg} / \mathrm{kg}\right.$ bodyweight/day) and should continue on the same regardless whether reactions occur.

An important implication of this policy is that para-medical workers should be better equipped to cope with reactions. They should be trained in the use of steroids and they should have clofazimine (Lamprene) freely at their disposal as an alternative to dapsone.

\section{Irregular Treatment}

Irregular treatment may be due to several factors, the most important being the leprosy worker himself who is often not aware of the fact that once he has started treating a lepromatous patient with DDS, he has taken a heavy personal responsibility upon himself: to see that that patient will receive uninterrupted full dose therapy throughout the duration of his illness. Conversely, every leprosy worker should realise that inadequate treatment of lepromatous patients during the first years may lead much later to sulphone resistance which cannot be undone by subsequently resorting to uninterrupted full dose therapy.

In the treatment of leprosy with its psychological and social overtones the relationship between patient and doctor or paramedical worker plays a crucial part. On the side of the patient confidence is a key factor in sustaining regular treatment. On the side of the leprosy worker this presumes professional skill, e.g. in coping with reactions, ulcers and deformities, personal interest in the patient which means willingness to spend time with him, perseverance and imagination, e.g. in setting up a postal DDS service for patients who are unable to collect their medicines at the appointed time.

Another important reason for irregular treatment viz. discontinuation of DDS during episodes of reaction has already been discussed.

Finally treatment may be interrupted because drugs like clofazimine or even dapsone are not readily available on account of production problems or import restrictions. The leprosy worker must be given his tools and nothing is more undermining to his morale than the absence of essential drugs. It is a tragedy when economical factors are responsible for the emergence of sulphone resistance with all its consequences to the patient and the community. Surely it ought to be possible to find a solution for such situations on a national or international level.

\section{Research}

While regular full dose therapy with DDS will reduce the risk of sulphone resistance in lepromatous patients, it will not completely exclude it, because M. leprae throws off drug-resistant mutants. There is also the possibility of a primary infection with DDS-resistant strains of M. leprae. For these reasons monotherapy with dapsone of lepromatous patients can no longer be considered

to be adequate and studies of drug combinations in the treatment of leprosy deserve high priority.

Another subject which urgently needs to be investigated is the prevalence of sulphone resistance in various countries. Central registration of suspected cases may give valuable information regarding the risk of sulphone resistance in relation 
to dosage and duration of treatment with DDS. This means that all leprosy workers should be constantly on the watch for such cases and have a good knowledge of the clinical signs of resistance, e.g. they should be aware that lepromatous nodules on the sclera, when seen in a patient on treatment with DDS almost always indicate resistance (Ross, 1976). They also should have a good knowledge of the interpretation of bacteriological findings (BI, MI). To assess accuracy of reported cases facilities may have to be arranged for mouse foot-pad inoculation of sample biopsies.

\section{Conclusion}

Sulphone resistance has emerged because of inadequate treatment of lepromatous patients. Therefore, it might be argued that unless one is certain that a lepromatous patient can be properly treated throughout the whole period of his disease, it would be wiser not to accept him for treatment at all! For once a patient has developed sulphone resistance he has become not only a therapeutic problem but a public health problem as well. Incidental treatment of lepromatous patients in hospitals or by private practitioners, and leprosy control programmes with a high rate of defaulters are likely to do more harm than good. Initially they may seem to be fairly successful but this is only because $M$. leprae is such a slow growing organism. Sooner or later, after a period of 5-20 years, sulphone resistance will catch up with us and we may find ourselves facing a greater problem than at the onset. Experience with tuberculosis and malaria makes it clear that in leprosy control programmes there is no room for light-hearted optimism. The implication for large scale treatment schemes is that the emphasis has to shift from quantity to quality. The population for whom a paramedical worker is responsible may have to be reduced and it may even become necessary to leave certain areas temporarily untouched in order to cover strategic areas properly. Only as individual and mass treatment increase in depth and effectiveness will hope be restored that leprosy can be controlled and eventually eradicated.

\section{References}

Pearson, J. M. H., Pettit, J. H. S. and Rees, R. J. W. (1968). The management of suspected sulphone resistant leprosy. Ninth Int. Lepr. Congr. (abstracts) 110.

Rees, R. J. W. (1968). Studies on drug resistance in leprosy using the mouse foot-pad infection. Ninth Int. Lepr. Congr. (abstracts) 102.

Ross, W. F. (1976). Essentials of Leprosy, Additional Notes. A.L.E.R.T., Addis Ababa. 